\title{
Development and Assessment of a Backward Facing Steps Planar Rocket Nozzle
}

\author{
Mandour $^{1}$ and Shaaban Abdallah ${ }^{2^{*}}$ \\ ${ }^{1}$ Ph.D. Candidate, Department of Aerospace Engineering, University of Cincinnati, OH 45221, USA \\ ${ }^{2}$ Professor, Department of Aerospace Engineering, University of Cincinnati, OH 45221, USA
}

*Corresponding author: Shaaban Abdallah, Department of Aerospace Engineering, University of Cincinnati, OH 45221, USA, E-mail: shaaban.abdallah@uc.edu

Received date: April 10, 2014; Accepted date: June 06, 2014; Published date: June 16, 2014

Copyright: (c) 2014 Mandour et al, This is an open-access article distributed under the terms of the Creative Commons Attribution License, which permits unrestricted use, distribution, and reproduction in any medium, provided the original author and source are credited.

\begin{abstract}
Large Rocket engine nozzles performance deteriorate because of asymmetric flow separation that lead to side loads. The dual-bell nozzle is a promising two operating modes nozzle which consists of a base nozzle with low area ratio and a nozzle extension with high area ratio. Although the dual-bell nozzle improves the performance of large rocket engines, it suffers from side loads that occur during the transition between the two operating modes. This paper presents and assess a new nozzle that consist of backward facing steps to minimize/eliminate side loads. The backward facing steps geometry guarantees fixed stable local separation at the steps edges at all altitudes, thus eliminates the possibility of side loads as the rocket ascends. The assessment of the nozzle performance is carried out using CFD simulation of turbulent Navier-Stokes equations solver (fluent 14.5). The computed results for both dual-bell and backward facing steps nozzles are compared with the dual-bell nozzle experimental data.
\end{abstract}

Keywords: Grid sensitivity; Computed pressure; Dual-bell nozzle; Asymmetric flow

\section{Introduction}

Classical bell nozzles that have fixed expansion area ratio cannot yield the maximum performance along a whole rocket trajectory. At low altitude, the nozzle experience asymmetric flow separation which generates heavy side loads. Among new nozzle concepts, the dual-bell nozzle is one of the most promising choices for improved rocket engine performance. The dual- bell nozzle is a two-mode altitude adaptive nozzle which consists of a base nozzle with low area ratio and a nozzle extension with high area ratio. At the junction between the base nozzle and the nozzle extension, a discontinuity exists in the slope of the nozzle (wall inflection). In low altitudes, a controlled flow separation occurs at the wall inflection leading to lower nozzle exit area (base nozzle area) which increases thrust gain and guarantees lower side loads. Experimental tests [1] on a subscale dual-bell nozzle were carried out to investigate the value of the side loads generated during transition from low to high altitude. It has been found in ref [1] that, although the transition duration is very short, typically in order of milliseconds, the separation point showed a significant asymmetry with an order of magnitude of the nozzle throat radius. A hot full-scale dual-bell nozzle test was carried out [2] to clarify why attention has to be paid to the onset of side loads during transition. The time interval during which the separation point lies inside the inflection region indicating the duration of the side loads during transition which was calculated to be approximately 11 seconds.

In this paper, we are interested in studying planar nozzles and developing a new backward facing steps nozzle. A literature survey for planar nozzles showed limited progress in this area.

In ref [3-6], numerical and experimental studies are carried out for conical and dual-bell planar nozzles. In ref $[3,4]$ a planar dual bell nozzle has been tested experimentally and numerically under different flow conditions. In the numerical study the difference in the separation position between two-dimension and three-dimension calculations was shown to be about 2-3\%. In ref [5] two dimensional simulations are carried out for a conical planar nozzle and the results for the wall pressure distribution and the flow structure at different pressure ratios compared well with the experimental data of ref [6].

We developed a new planar nozzle to provide an altitude adaptation reduction/elimination of side loads. The new nozzle consists of a conventional bell nozzle base with low area ratio, identical to the dualbell nozzle, and a nozzle extension geometry that consists of backward facing steps. The goal of the backward facing steps geometry is to create controlled separation that guarantees lower side loads at each altitude during rocket ascend.

\section{Analysis}

Two-dimensions numerical solutions for the dual-bell nozzle and backward facing steps nozzle shown in figure. 1 are obtained from solving the unsteady Reynolds-averaged Navier-Stokes equations using an explicit second-order time-and-space accurate schemes (Fluent code). The time dependent numerical simulation is carried out for time; $\mathrm{t}=0-9$ seconds corresponding to nozzle pressure ratio; $\mathrm{PR}=24.24-45.41$. In the numerical simulation, the total pressure in the combustion chamber (nozzle inlet) is kept constant while the ambient pressure is varied with altitude as follows:

$$
\mathrm{P}=101325 \mathrm{e}^{(-\mathrm{H} / 7000)}
$$

Where $\mathrm{P}$ is the ambient pressure and $\mathrm{H}$ is the altitude. Assuming that the rocket is flying with constant velocity equal $500 \mathrm{~m} / \mathrm{s}$, the ambient pressure related to the time $(t)$ can be written as

$$
\mathrm{P}=101325 \mathrm{e}^{\left(-500^{*} \mathrm{t} / 7000\right)}
$$

As the ambient pressure equivalent to $\mathrm{PR}=24.24$ ( $\mathrm{t}=0$ seconds), the pressure equation as a function of time is modified by curve fitting to get the ambient pressure-time relationship used in this study:

$$
\mathrm{P}=38411 \mathrm{e}^{\left(-0.071^{*} \mathrm{t}\right)}
$$




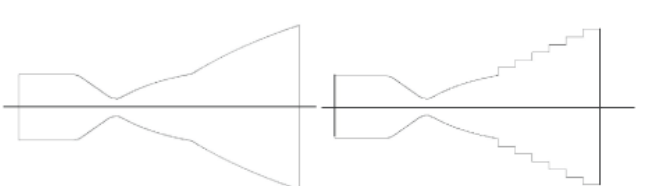

Figure 1: Geometries of dual-bell and backward facing steps nozzles.

\section{Grid sensitivity analysis}

A grid independent study was performed by solving the governing equations for different grid densities ranging from $168,000-650,000$ elements. The flow parameters at the nozzle exit for $\mathrm{PR}=24.24$ show that the maximum difference between the results of the 348,000 and 650,000 grid elements is less than $0.4 \%$. Therefore, the grid with 348,000 elements is used in the present results.

\section{Numerical Results and Discussions}

First, the numerical procedure is validated by comparing the computed results of the dual-bell nozzle with the experimental data from ref [7] for different vertical planes (TB, TD, TC, and BC) in the planar dual-bell nozzle. These planes are vertical planes between the two side walls of the planar nozzle. TB and TD are the top of the planes $\mathrm{B}$ and $\mathrm{D}$ respectively, while $\mathrm{TC}$ and $\mathrm{BC}$ are the top and the bottom of plane C. Figures 2 and 3 shows the pressure distribution over the nozzle wall at PRs equal 15.5 and 24.24 respectively. The computed results for pressure and location of the flow separation are in good agreement with the experimental data, thus the procedure is validated.

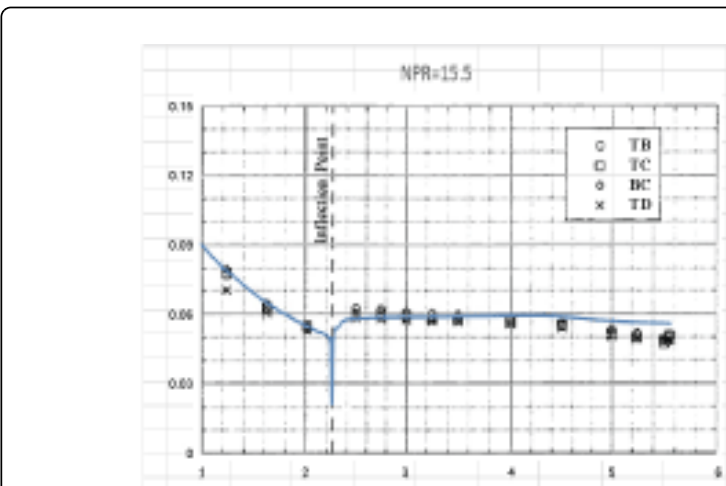

Figure 2: Computed pressure distribution over the nozzle wall compared with experimental data for NPR=15.5.

Second, numerical solutions are obtained for both the backward facing steps nozzle and the dual-bell nozzle for time; $t=0-9$ seconds for comparison. Figures 4-7 show the wall pressure distribution for the dual-bell nozzle at $\mathrm{t}=0,4,5$, and 6 seconds. Figure. 4 shows that the separation point is located at the end of the base nozzle at $P R=24.24$. In figures 5-7 it can be seen that as the pressure ratio increases the flow separation point leaves the wall inflection point and moves downstream towards the nozzle exit. In the same figures, an asymmetric flow separation over the upper and the lower nozzle walls is observed. It starts at $\mathrm{t}=4$ seconds and the maximum side load occurs at $\mathrm{t}=5$ seconds and equal to $12.6 \mathrm{~N}$. Figures (8-11) show the wall pressure distributions for the backward facing steps nozzle at $\mathrm{t}=0,4,5$, and 6 seconds. Fig (8) shows that the first separation point is located at the first backward facing step edge for $\mathrm{PR}=24.24$.

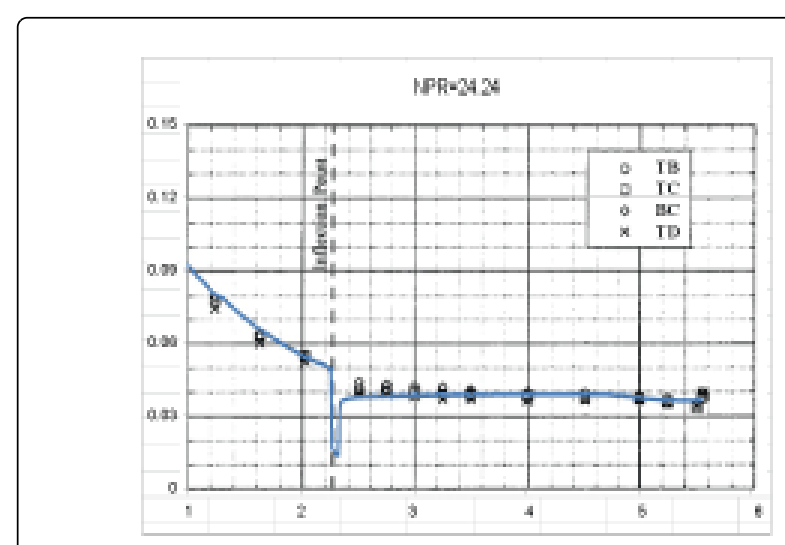

Figure 3: Computed pressure distribution over the nozzle wall compared with experimental data for $\mathrm{NPR}=24.24$.

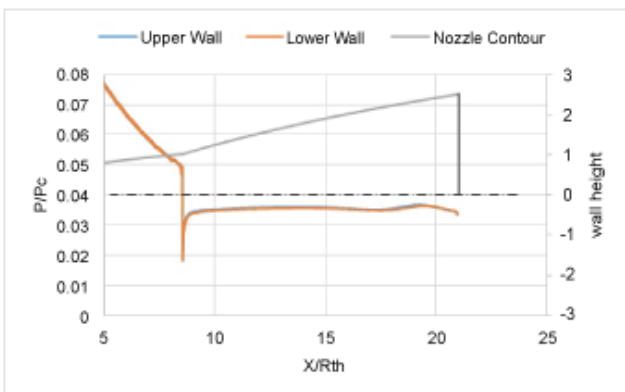

Figure 4: Wall pressure distribution for dual-bell nozzle at $\mathrm{t}=0$ seconds.

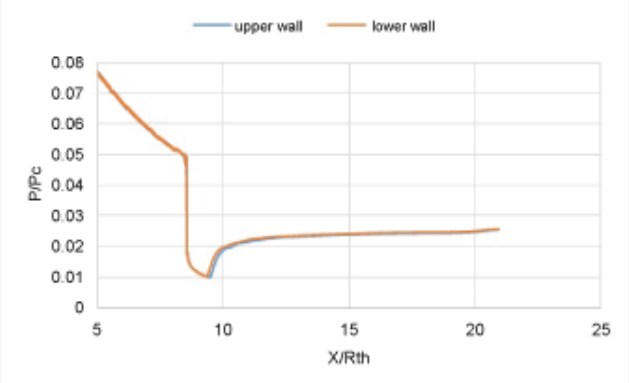

Figure 5: Wall pressure distribution for dual-bell nozzle at $\mathrm{t}=4 \mathrm{sec}$ seconds.

It is shown in figures (9-11) that the separation point is maintained at different altitudes at the backward facing steps as the pressure ratio increase with time (altitude). A strong pressure gradient occurred at each backward facing step where the flow separation exist. That creates a stable position for the flow separation points for a wide range of 
Citation: Mandour, Shaaban Abdallah (2014) Development and Assessment of a Backward Facing Steps Planar Rocket Nozzle . Glob J Tech

operating pressure ratios. It can also be noticed that the pressure distributions over the upper and lower nozzle walls are quite similar to each other's which indicate a symmetrical flow separation and low side load forces for different pressure ratios. Table 1 shows the comparison of the side load magnitudes between the backward facing steps nozzle and the dual-bell nozzle at different PRs.

\begin{tabular}{|l|l|l|l|l|}
\hline \multirow{2}{*}{ Time } & \multirow{2}{*}{ PR } & \multicolumn{2}{|l|}{ Side load [N] } \\
\cline { 3 - 5 } & & \multicolumn{2}{|l|}{ Backward facing } \\
\cline { 3 - 5 } & & \multicolumn{2}{|l|}{ Dual-bell nozzle } \\
\hline 0 & 24.24 & 0.501 & 0.3165 & -36.83 \\
\hline 4 & 32.202 & 4.129 & 0.4167 & -89.91 \\
\hline 5 & 34.572 & 12.682 & 0.3046 & -97.6 \\
\hline 6 & 37.116 & 5.08 & 0.2389 & -95.3 \\
\hline
\end{tabular}

Table 1: Comparison of side load magnitudes between backward facing steps nozzle and dual-bell nozzle.

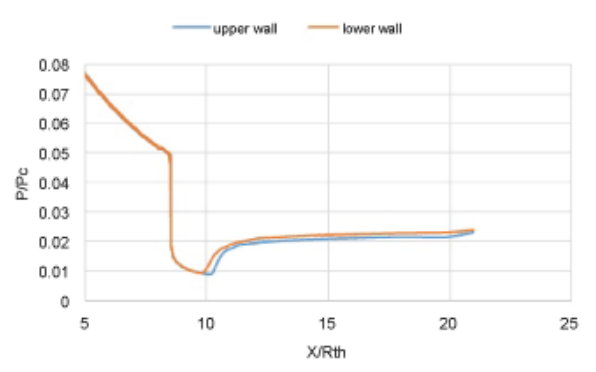

Figure 6: Wall pressure distribution for dual-bell nozzle at $t=5$ seconds.

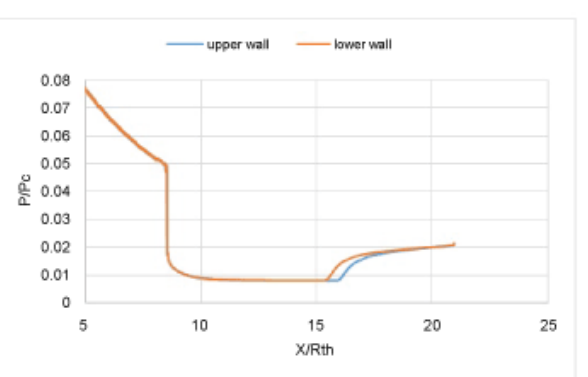

Figure 7: Wall pressure distribution for dual-bell nozzle at $\mathrm{t}=6$ seconds.
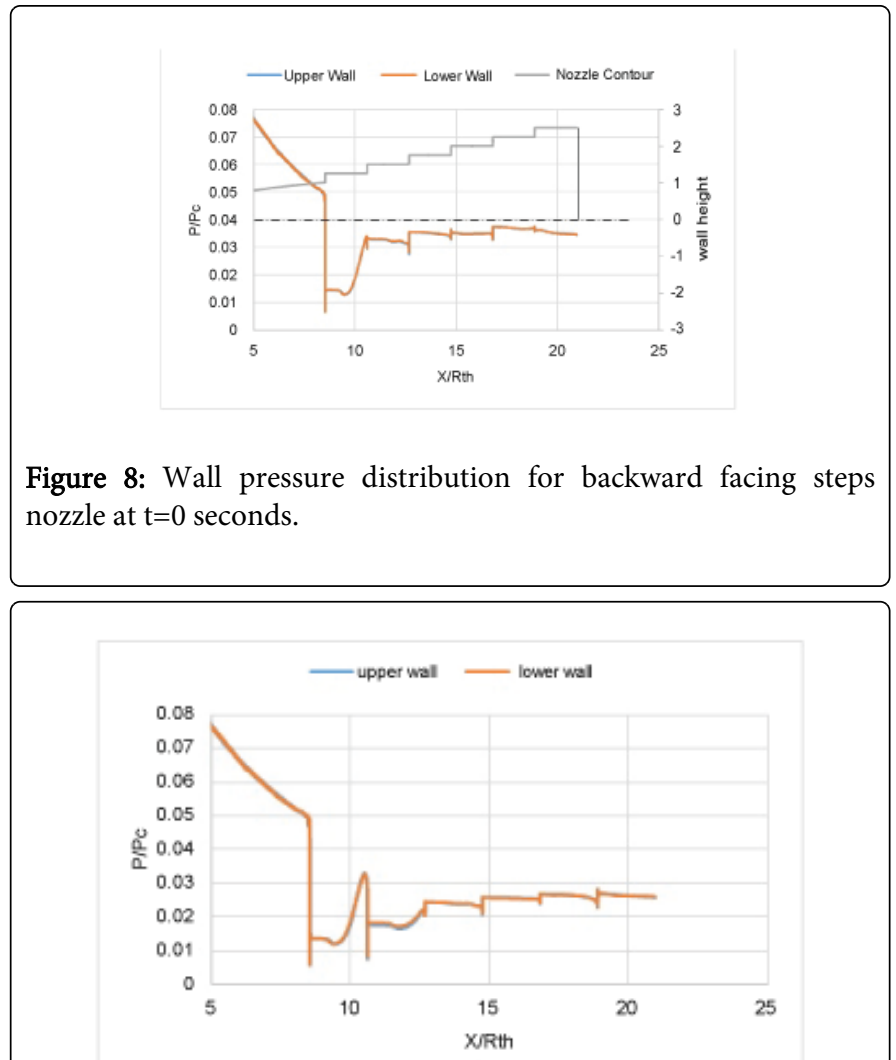

Figure 9: Wall pressure distribution for backward facing steps nozzle at $\mathrm{t}=4$ seconds.

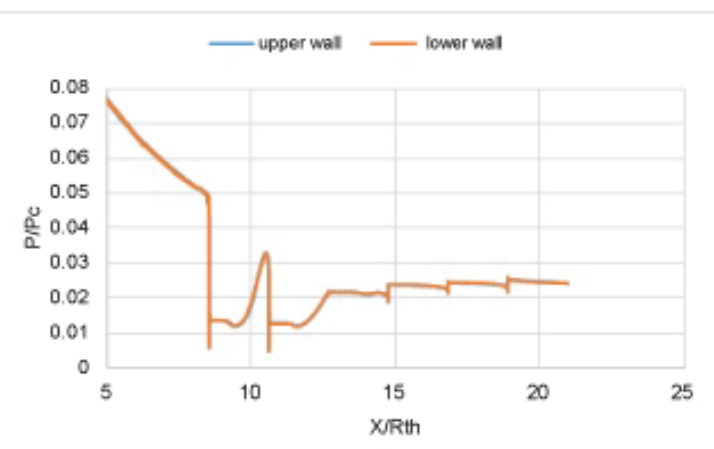

Figure 10: Wall pressure distribution for backward facing steps nozzle at $\mathrm{t}=5$ seconds. 


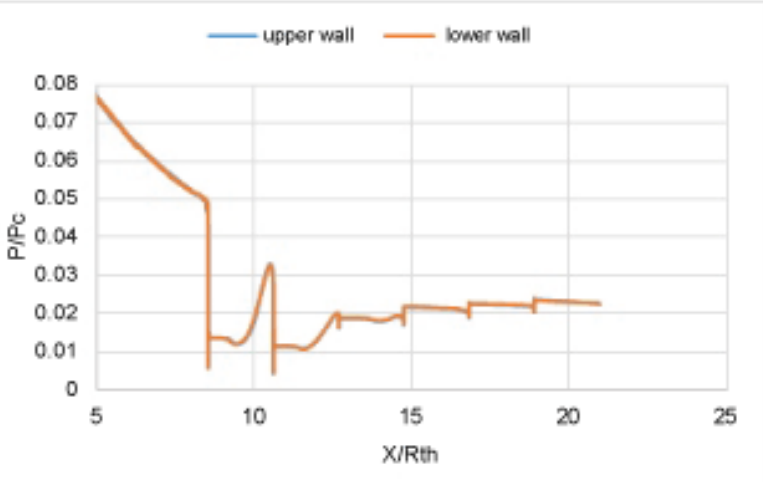

Figure 11: Wall pressure distribution for backward facing steps nozzle at $\mathrm{t}=6$ seconds.

It is important to mention here that the study of ref [8] examined three families of profiles of the dual-bell nozzle extension: (1) profile with increasing wall pressure, (2) profile with constant wall pressure, and (3) profile with decreasing wall pressure. A time-accurate simulation in ref [8] shows that an important reduction of the transition time (time of peak side loads during transition) can be achieved by using extension shapes featuring positive values of pressure gradient. In the backward facing steps nozzle, the pressure on the backward facing steps edges has overall positive pressure gradient as shown in figure (12). This finding validates the promise of the backward facing steps nozzle to reduce/eliminate side loads.

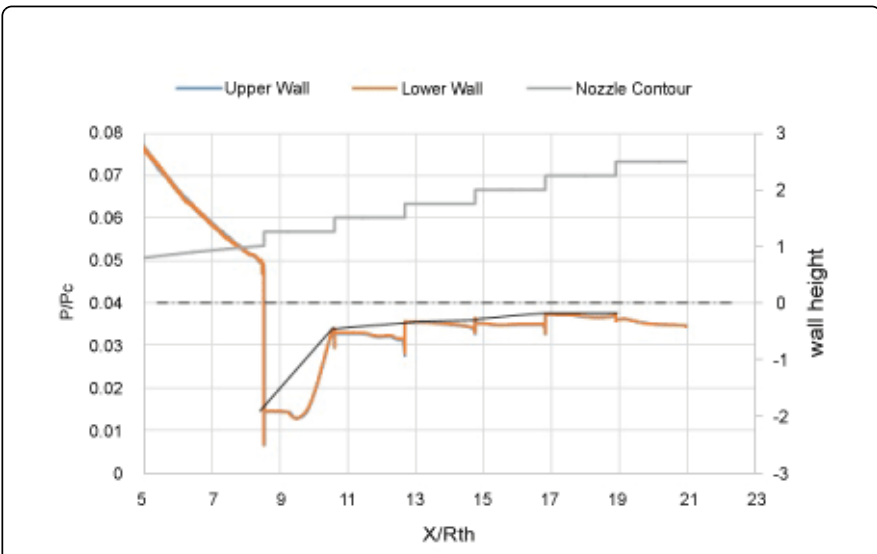

Figure 12: Wall pressure distribution for backward facing steps nozzle at $\mathrm{t}=0$ seconds.

\section{Conclusions}

A new backward facing steps nozzle is developed and assessed numerically for a planar nozzle geometry by comparison with dualbell nozzle experimental data and computational results. The numerical solutions are obtained by solving the Reynolds averaged Navier-Stokes equations using Fluent code. From the numerical solutions, an asymmetric flow separation and wall pressure distributions are observed in the dual-bell nozzle at different pressure ratios which led to high side loads. On the other hand, the results of the backward facing steps nozzle show a symmetric stable flow separation with slightly different wall pressure distributions that led to small side loads. The maximum side load of the dual-bell nozzle is reduced by $97.59 \%$ by backward facing steps. In the backward facing steps nozzle, the pressure on the backward facing steps edges show overall positive pressure gradient which validates the promise of the backward facing steps nozzle to reduce/eliminate side loads. It is important to state here that no optimization is carried out for the backward facing steps geometry which may have the potential for total elimination of the side loads.

\section{References}

1. Chloe Genin and Ralf Stark, (2011), Side Loads in Subscale Dual Bell Nozzles, Journal of Propulsion and Power, 27(4), pp. 828-837.

2. Emanuele Martelli, Francesco Nasuti and Marcello Onofri, (2007), Numerical Parametric Analysis of Dual-Bell Nozzle Flows, AIAA Journal, 45 (3), pp.640-650.

3. C. Genin, R. Stark and O. Haidn (2011) Experimental and Numerical Study of Dual Bell Nozzle Flow, 4th European Conference for Aerospace Sciences, 5, pp. 363-376.

4. Chloe Genin, Andreas Gernoth, and Ralf Stark (2013) Experimental and Numerical Study of Heat Flux in Dual Bell Nozzles, Journal of Propulsion and Power, 29(1), pp.21-26.

5. Q. Xiao and H. M. Tsai, (2007). Numerical Investigation of Supersonic Nozzle Flow Separation, AIAA Journal, 45(3), pp.532-541.

6. Dimitri Papamoschou and Andreas Zill, (2004). Fundamental Investigation of Supersonic Nozzle Flow Separation, 42nd AIAA Aerospace Sciences Meeting and Exhibit, Reno, Nevada.

7. Jeff Yu-Chin Lin (1997). A Numerical Analysis of a Two Dimensional Dual-Bell Nozzle, M.Sc. Thesis, Department of Mechanical and Aerospace Engineering, University of Alabama, Huntsville, p.176.

8. Francesco Nasuti, Marcello Onofri and Emanuele Martelli (2005). Role of Wall Shape on the Transition in Axisymmetric Dual-Bell Nozzles, Journal of Propulsion and Power, 21(2), 243-250. 